\title{
Morphofunctional MR in the Diagnosis and Follow-Up of Cerebral Gliomas
}

\author{
Tommaso Scarabino, Alberto Maggialetti, Saverio Pollice, \\ Angela Lorusso, Gilda Morlino, Michela Capuano, \\ and Teresa Popolizio
}

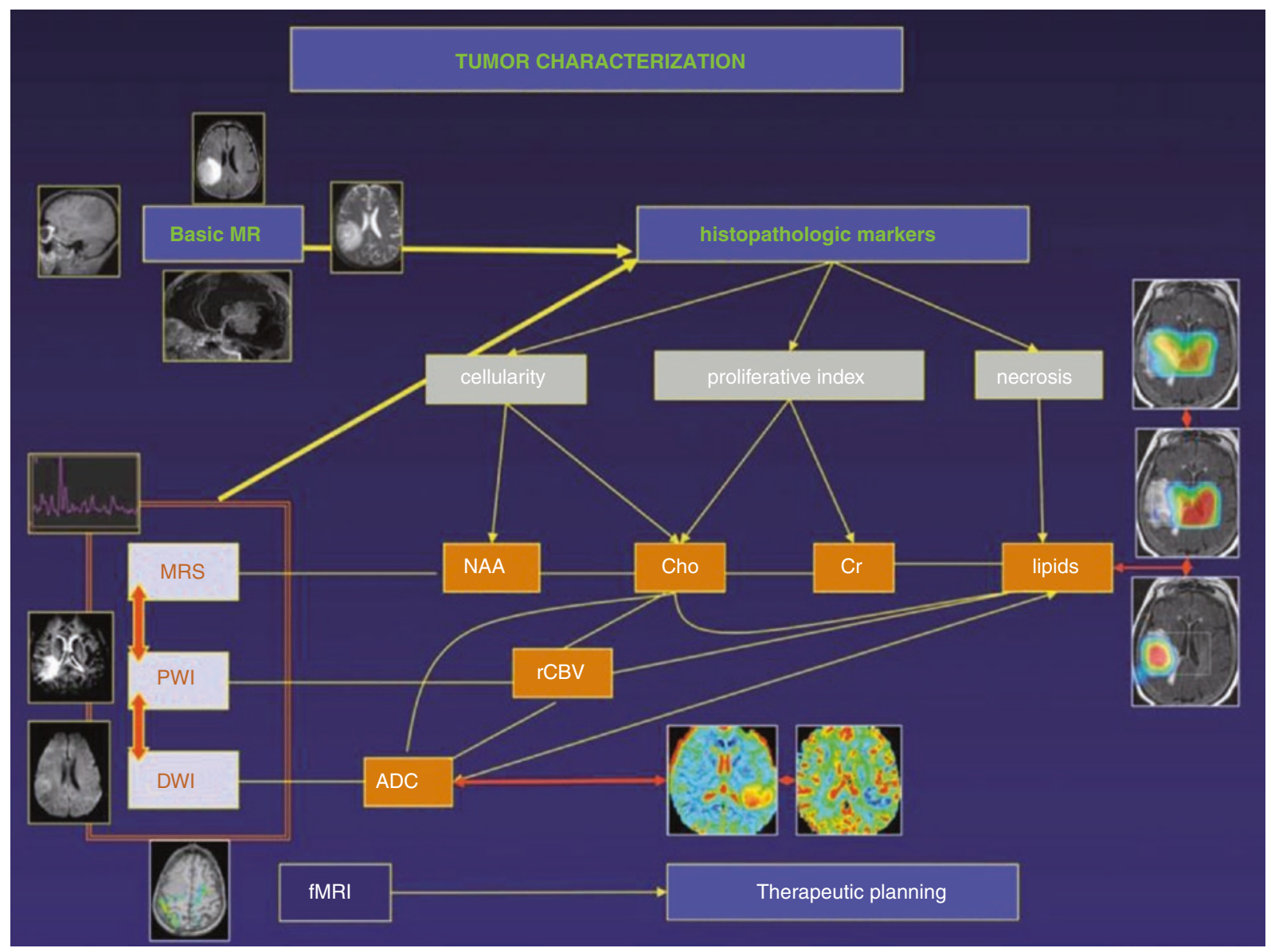

Fig. 55.1 Morphologic MR imaging without and with contrast medium is the examination of choice for the characterization of tumors in both diagnosis and follow-up. A thorough analysis of the radiologic signs, the comparison with the patient's clinical information, and the therapy performed can lead to a correct diagnosis. However, some cases are difficult to diagnose because of the extreme tissue heterogeneity and the frequent coexistence of different pathologic patterns. The morphologic examination therefore may require the addition of advanced MR techniques, i.e., functional MR (spectroscopy, diffusion, perfusion, cortical activation), which enable a neuropathologic study in vivo. The functional and physiologic data on cell metabolism, as well as hemodynamic and diffusion parameters, provide a biologic interpretation of the heterogeneity and complexity typical of these tumors, thus improving the diagnostic accuracy, sensitivity, and specificity of MR and enabling a more precise and comprehensive diagnosis for the surgeon
T. Scarabino $(\bowtie) \cdot$ S. Pollice $\cdot$ A. Lorusso $\cdot$ M. Capuano Department of Radiology, "L. Bonomo" Hospital, Andria, BT, Italy

A. Maggialetti

Ricerche Radiologiche Center, Molfetta (Ba), Italy

\section{G. Morlino}

Department of Radiology, "Di Venere" Hospital, Bari, Italy

T. Popolizio

Department of Neuroradiology, Scientific Institute "Casa Sollievo della Sofferenza", San Giovanni Rotondo (Fg), Italy 